\title{
CandyCollect: At-home saliva sampling for respiratory pathogen capture
}

Ulri N. Lee, ${ }^{*, 1}$ Xiaojing Su, ${ }^{*, 1}$ Damielle L. Hieber, ${ }^{1}$ Wan-chen Tu, ${ }^{1}$ Anika M. McManamen, ${ }^{1}$ Meg G. Takezawa, ${ }^{1}$ Grant W. Hassan, ${ }^{1}$ Tung Ching Chan, ${ }^{1}$ Karen N. Adams, ${ }^{2}$ Ellen R. Wald, ${ }^{3,4}$ Gregory P. DeMuri, ${ }^{3,4}$ Erwin Berthier, ${ }^{1}$ Ashleigh B. Theberge ${ }^{\sharp 1,5}$ and Sanitta Thongpang, ${ }^{\sharp 1,6}$

${ }^{1}$ Department of Chemistry, University of Washington, Seattle, WA, USA

${ }^{2}$ Institute of Translational Health Sciences, School of Medicine, University of Washington, Seattle, WA, USA

${ }^{3}$ American Family Children's Hospital, Madison, WI, USA

${ }^{4}$ Department of Pediatrics, University of Wisconsin School of Medicine and Public Health, Madison, WI, USA

${ }^{5}$ Department of Urology, University of Washington School of Medicine, Seattle, WA, USA

${ }^{6}$ Department of Biomedical Engineering, Faculty of Engineering, Mahidol University, Nakorn

Pathom, Thailand

*These authors contributed equally to this work.

\#Co-corresponding authors: sanitta@uw.edu, abt1@uw.edu

\begin{abstract}
Streptococcus pyogenes is a major human-specific bacterial pathogen and a common cause of a wide range of symptoms from mild infection such as pharyngitis (commonly called strep throat) to life-threatening invasive infection. Traditional methods for diagnosis include collecting a sample using a pharyngeal swab, which can cause discomfort and even discourage adults and children from seeking proper testing and treatment in the clinic. Saliva samples present an alternative to pharyngeal swabs. To improve the testing experience for strep throat, we developed a novel lollipop-inspired sampling platform (called CandyCollect) to capture bacteria in saliva. The device can be used in clinics or in the home and shipped back to a lab for analysis, integrating with telemedicine. CandyCollect is designed to capture bacteria on an oxygen plasma treated polystyrene surface embedded with flavoring substances to increase the children's compliance and inform the required time to complete the sampling process. In addition, the open channel structure prevents the tongue from scraping and removing the captured bacteria. We also demonstrated that flavoring substances did not affect bacterial capture, and that the device has a shelf life of at least 2 months (with experiments ongoing to extend the shelf life). We performed a usability study with 17 participants who provided feedback on the device design and the dissolving time of the candy. This technology and advanced processing techniques, including polymerase chain reaction (PCR), will enable user-friendly and effective diagnosis of streptococcal pharyngitis.
\end{abstract}

\section{Introduction}

Acute respiratory infections are common with children averaging 3-5 upper respiratory infections per year, some of which may lead to additional complications if left untreated. ${ }^{1}$ In developing countries, these illnesses can be more severe, with gaps in healthcare increasing the risk of morbidity and mortality. ${ }^{2}$ Additionally, even in developed countries, people who have limited access to clinics, such as in rural areas, or those without insurance may also be unable to receive appropriate diagnosis and treatment. Here we develop a new sample collection platform for a common bacterial infection, group A streptococcal (GAS) pharyngitis, also known as strep throat, 
caused by Streptococcus pyogenes. GAS pharyngitis is commonly treated with antibiotics; it can lead to suppurative complications (acute otitis media, sinusitis, retro- and peritonsillar abscess, cervical adenitis), rheumatic fever, and organ damage if left undiagnosed and untreated.

The gold standard method for diagnosis of GAS pharyngitis is swabbing the posterior pharynx and testing with a rapid antigen detection test (RADT); standard culture is performed if the RADT is negative. ${ }^{3,}{ }^{4}$ However, culture is time-consuming. ${ }^{5}$ Currently, there are numerous advanced techniques for the detection of $S$. pyogenes, such as polymerase chain reaction (PCR), ${ }^{6,7}$ multiplex $\mathrm{PCR},{ }^{8}$ impedimetric biosensors, ${ }^{9}, 10$ touch spray-mass spectrometry, ${ }^{11}$ and commercial test kits (e.g., BinaxNOW ${ }^{\circledR}$ Strep A Test; OSOM ${ }^{\circledR}$ Ultra Strep A Test; BD Chek ${ }^{\mathrm{TM}}$ Group A Strep; QuickVue In-line Strep A Test). Some commercially available at-home GAS test kits, such as ezlevel Strep A test, (C) ezleveltests, rely on the parent swabbing the throat which is not used as the standard of care because it does not produce reliable results. ${ }^{12}$ Even in a clinical setting it can be challenging to obtain a high-quality swab sample of the posterior pharynx including the tonsils due to child resistance and discomfort. ${ }^{13}$ Children may even refuse to use or be noncompliant with oral sample collection, which can lead to skewed results. ${ }^{13}$ Recently, saliva samples have been shown to be an alternative to pharyngeal swabs for respiratory disease diagnosis. ${ }^{14,15} \mathrm{~A}$ recent study of GAS in the saliva of children used swabs to absorb saliva and PCR for detection; 19 of 20 patients with previously confirmed GAS pharyngitis tested positive. ${ }^{15}$ In the present work, we aim to further improve the patient experience and establish a salivary sampling open-fluidic device for $S$. pyogenes capture, which can be used comfortably in a clinic or home setting for adults and children.

Our open-fluidic sampling device, called the CandyCollect and inspired by a lollipop, includes a small polystyrene stick with one end coated in isomalt and fruit flavoring candy. We aimed to engineer a device that is both efficient at capturing bacteria in saliva samples and decreases the discomfort experienced in traditional swabbing methods. We demonstrate that the mass of the candy can be used to inform the sampling time, effectively using the disappearance of the flavoring as a built-in timer. Additionally, we conducted in vitro experiments involving the target pathogen, $S$. pyogenes, to investigate its adherence to the device and the effect of sample evaporation on elution for further sample processing procedures. We have successfully demonstrated that the open-fluidic channel prevents the tongue from scraping the surface and retains the bacteria within the channel, demonstrating that our device can capture the pathogen and accumulate more bacteria over time without disturbance from external factors. To assess the usability and feasibility of this device, we sent CandyCollects to 17 participants across the country to record the person-to-person differences in candy dissolving time and user feedback. With the growing need for decentralized medicine and telehealth solutions, our device provides an accessible sampling method for more versatility in at-home and clinical settings.

\section{Results and Discussion}

Noncompliance with diagnostic testing in children affects accuracy and efficacy of diagnosis for upper respiratory infections, and the inability to access a medical professional also prevents children from receiving treatment. When left untreated, diseases such as strep throat can have severe consequences such as rheumatic fever, organ damage, secondary infections, and death. ${ }^{1,2}$, 16 To optimize the diagnosis of streptococcal pharyngeal infections we have developed a saliva sampling device that takes design inspiration from a classic childhood treat, a lollipop. 
a)

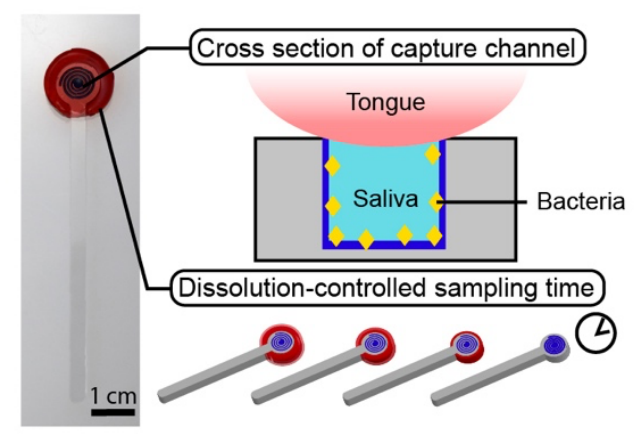

b)

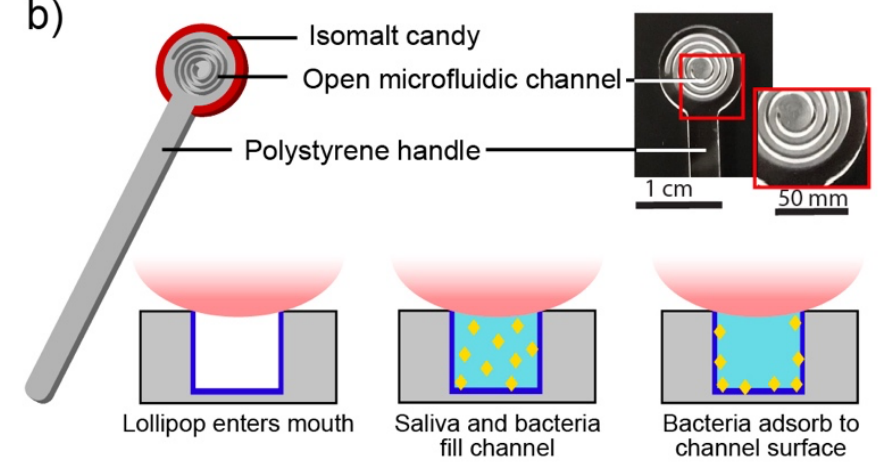

Figure 1. The CandyCollect is a lollipop-inspired at-home saliva collection and bacteria sampling device for S. pyogenes, the bacteria that causes pharyngeal GAS infection (commonly called strep throat). a) The open-fluidic channel in the CandyCollect captures the bacteria from saliva, and the candy flavoring is a built-in timer for sampling time (i.e., dissolving time of the candy). The openfluidic channel is designed to prevent the tongue from scraping the bottom of the channel and removing the collected bacteria, allowing bacteria to accumulate during the sampling time. b) The CandyCollect is composed of a polystyrene stick with a microfluidic channel and red isomalt candy. When the CandyCollect enters the mouth the channel fills with saliva and the bacteria adheres to the plasma treated surface of the channel.

CandyCollect was designed to comfortably fit in a child's mouth, mitigate any potential choking hazard by use of a thin candy coating rather than a large piece of candy, be compatible for use with common elution kits, such as ESwab 480C (Copan), and have optimal saliva exchange with the capture surface. The lollipop sampling device consists of a computer numerical control (CNC) milled polystyrene stick that is coated on the back and edges with isomalt candy. The face of the stick is not coated in candy and contains a plasma treated open-fluidic spiral channel for saliva and bacteria capture (Figure 1). Plasma treatment is known to facilitate cell adhesion on polystyrene surfaces. ${ }^{17-19}$ The aspect ratio of the channel was designed such that a tongue cannot scrape the bottom of the channel and remove captured bacteria. Isomalt, a sugar alcohol, was used for the candy because it has a low hygroscopicity (ERH of 88), meaning it will not absorb water until equilibrium relative humidity reaches $88 \%$ at $20^{\circ} \mathrm{C} .{ }^{20}$ which enables it to remain stable if packaged and stored for extended periods of time. Additionally, in the presence of oral bacteria, such as Streptococcus mutans, sucrose ferments and becomes turbid while isomalt and other polyols do not. ${ }^{20}$ Finally, isomalt has a glycemic index of two and is safe for diabetic patients. ${ }^{21}$ The candy also acts as a built-in timer for sampling time and ensures the patient keeps the device in their mouth for the optimal time for bacteria capture. When the candy flavoring disappears, the sampling is complete, and the patient can mail the CandyCollect back or schedule a pickup for analysis. With the amount of isomalt on the lollipop we can adjust for different sampling times required by other respiratory pathogens.

The ability to enhance or accumulate the pathogens of interest over time shows the future potential of CandyCollect to capture a range of pathogens that are present at different concentrations. Determining how much $S$. pyogenes the device can capture is important in determining if the device will be compatible with current quantification or rapid PCR methods such as qPCR or realtime-PCR. Additionally, evaluating if bacteria collection increases over time relies on tailoring the mass and surface area ratio of the candy to control the sampling time. In vitro, our device can 
a)

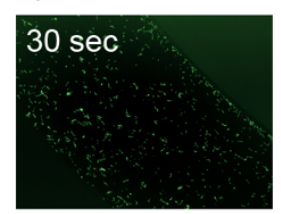

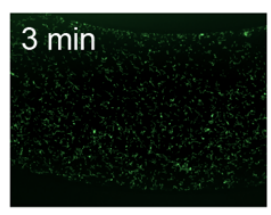

b) Channel prevents $S$. pyogenes from being scraped off Before scraping with cotton swab

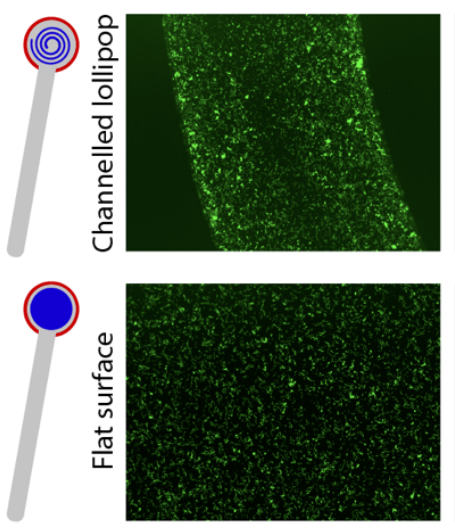

After scraping with cotton swab
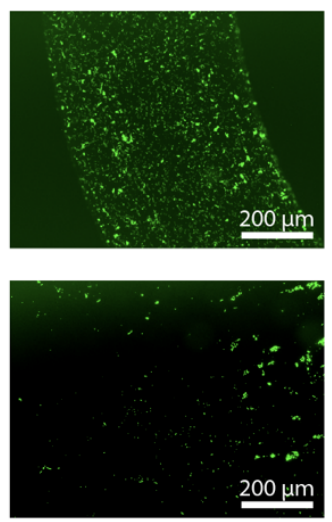

Figure. 2 S. pyogenes accumulates over time, and the channel geometry prevents loss of captured $S$. pyogenes. a) S. pyogenes captured in vitro on the lollipop accumulates over $30 \mathrm{sec}, 3 \mathrm{~min}$, and 10 min. b) S. pyogenes in vitro remains in the channel following scraping with a cotton swab (intended to represent the tongue scraping the device) compared to markedly reduced amounts in a lollipop without a channel (flat surface) of identical design and concentration of exposure to $S$. pyogenes. Note: $1 \times 10^{7} \mathrm{CFU} / \mathrm{mL}$ suspension was used in part a, and $1 \times 10^{9} \mathrm{CFU} / \mathrm{mL}$ suspension was used in part b (an incubation time of 10 min was used in b). Images are representative of 3 independent experiments, with duplicates and 4 images taken per replicate.

accumulate S. pyogenes over time and capture it in as little as 30 seconds (Figure 2a). Further, it was important to show that the channels prevented the tongue from scraping off any captured $S$. pyogenes (Figure $2 \mathrm{~b}$ ). A shallow circle was milled for the flat surface lollipop (Figure $2 \mathrm{~b}$, bottom row) which serves as a control because it has the same milled surface as the channel lollipop. A cotton swab was used to simulate the tongue and was scraped across the surface of both the flat and channeled lollipop. Fluorescence microscopy images indicate the channel protects the $S$. pyogenes from being scraped off as opposed to the flat surface which shows a marked decrease in S. pyogenes after scraping (Figure $2 \mathrm{~b}$ ).

Our device was designed to enable a high-quality, comfortable collection of pathogens from saliva in a home or clinical setting. We anticipate the devices to be stored in homes or clinics, thus it is highly likely the devices would be stored for extended periods of time before usage. It is known that over time plasma treated surfaces become more hydrophobic (water contact angle increases within 10 days and stabilizes with long storage time). ${ }^{22}$ To test whether the time-induced changes in the plasma treated surface of CandyCollect affect its ability to capture bacteria, we conducted shelf life experiments at room temperature. Fluorescence microscopy images show that there is no 


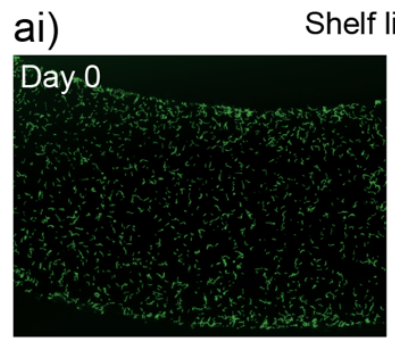

Shelf life test
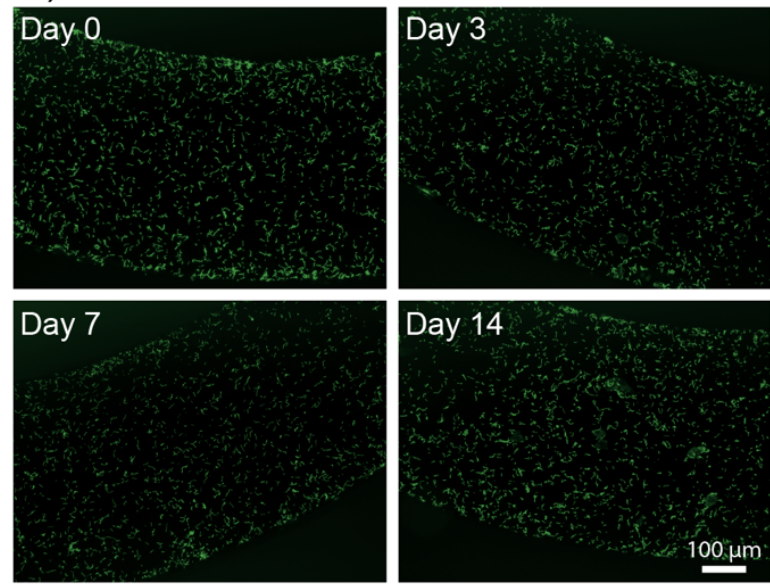

aii)
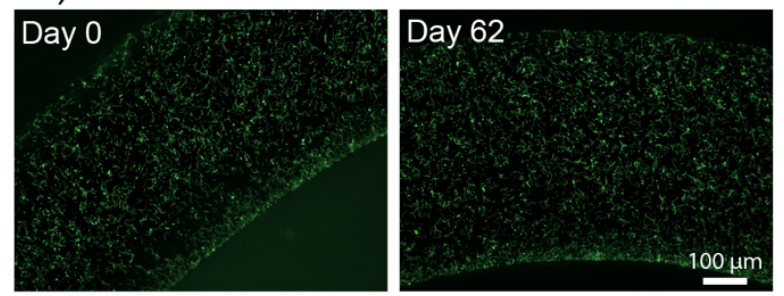

b) Dried sample
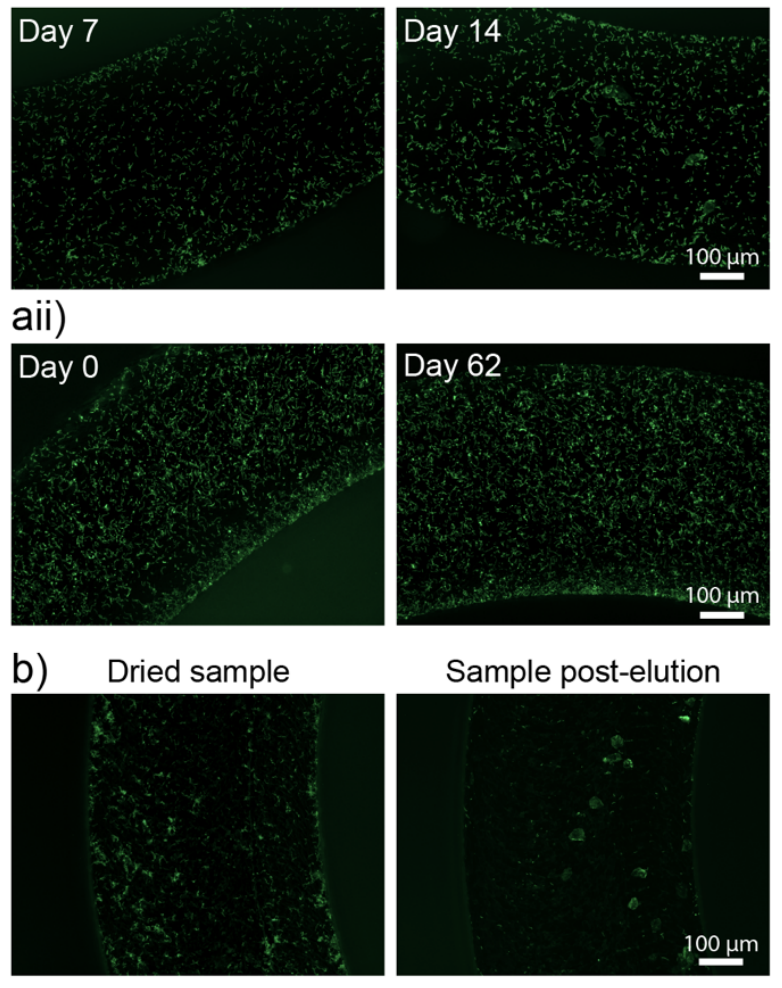

Sample post-elution

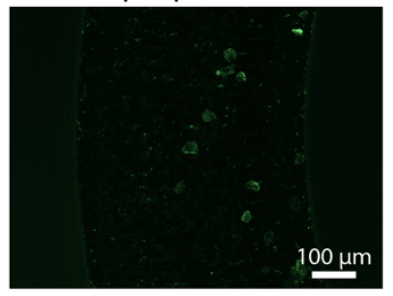

Figure 3. Shelf life tests demonstrate that CandyCollect effectively captures S. pyogenes after 2 months of storage. Devices were plasma treated and stored at room temperature for ai) 0 days (control group), 3 days, 1 week, 2 weeks, and aii) 0 day (control group) and 2 months before capturing the $S$. pyogenes in vitro. Fluorescence microscopy images indicate capture of $S$. pyogenes after 62 days of storage is similar to the control. b) Elution of $S$. pyogenes is successful in dried samples. Devices were left at room temperature until saliva evaporated, rehydrated with phosphate buffered saline, eluted with DNAzol, and imaged. Images indicate $S$. pyogenes could be eluted by the elution buffer, such as DNAzol. Note: in both a and b, S. pyogenes at a concentration of $1 \times 10^{9} \mathrm{CFU} / \mathrm{mL}$ was incubated on the CandyCollect device for 10 minutes. Images are representative of 3 independent experiments, with triplicates and at least 4 images taken per replicate.

noticeable difference observed in the images between 0 day (control group), 3 days, 1 week, and 2 weeks (Figure 3ai). An additional experiment was conducted to test two months of storage, which also showed the device was still able to capture $S$. pyogenes efficiently compared to the control (Figure 3aii). In the future, we plan to conduct shelf life experiments for lengths such as six months to one year. 
The saliva on the devices may evaporate in the time between sampling and analysis if they are being shipped back to the laboratory. It is important to note that we will not be culturing the bacteria from the CandyCollect device but rather eluting the bacteria from the device for qPCR analysis to detect the presence or absence of $S$. pyogenes. To evaluate whether or not the drying affects the ability to elute $S$. pyogenes, we left the devices to evaporate $(10 \mathrm{~min})$. Fluorescence microscopy images showed that the $S$. pyogenes captured on devices could still be eluted from the device for further experiments (Figure 3b). Preliminary experiments demonstrate that qPCR can be successfully performed on samples eluted from the CandyCollect device (data not shown); we will optimize the elution and qPCR procedures in future work when evaluating and optimizing the limits of detection of our system.

Multiple pathogens in human saliva have different typical concentrations, therefore; flexibility in dissolving time of the candy is desirable. To demonstrate flexibility in dissolving time, six CandyCollects with different masses of candy were created to determine how the mass and dimensions of the candy affect the dissolving time. One individual consumed three CandyCollects of each version and recorded the dissolving time (Figure 4a). Based on the results, the CandyCollects dissolved between less than one minute and nearly fifteen minutes, accommodating a range of sampling times that may be needed depending on the target bacteria. The full set of data including the mass and dimensions of the CandyCollects is available in Table S1. However, the CandyCollects are not limited to this range and can be made larger to accommodate sampling times longer than fifteen minutes.

a)

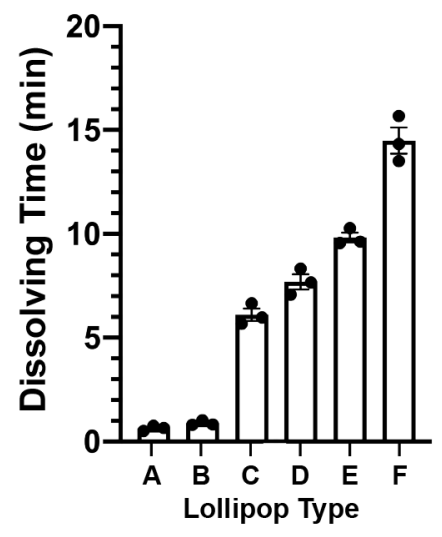

b)

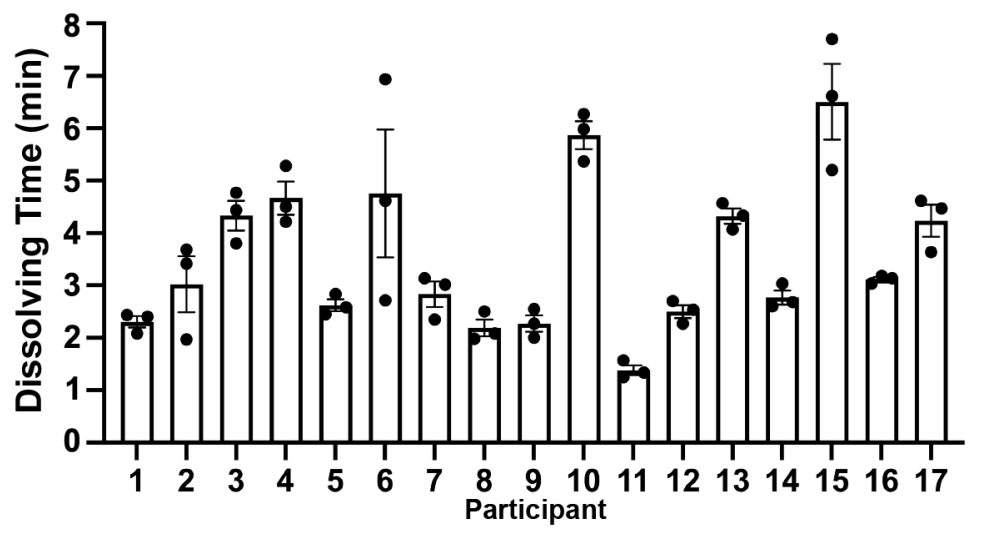

Figure 4. Lollipop dissolving time can be controlled by changing the mass and size of the lollipop. a) Six types of CandyCollect devices, each with a different mass and size of candy, were consumed by one individual. Bar graphs represent mean \pm SEM of $n=3$ CandyCollects. Please refer to Table $\mathrm{S} 1$ in the Supporting Information for lollipop mass and dimensions. b) A single size of CandyCollect devices was sent to 17 research participants for a usability test. The mass of the lollipop in $\mathrm{b}$ is $0.90-1.10 \mathrm{~g}$, with a diameter of $16 \mathrm{~mm}$, and thickness of $4 \mathrm{~mm}$. Bar graphs represent mean $\pm \mathrm{SEM}$ of $\mathrm{n}=3$ CandyCollects. 


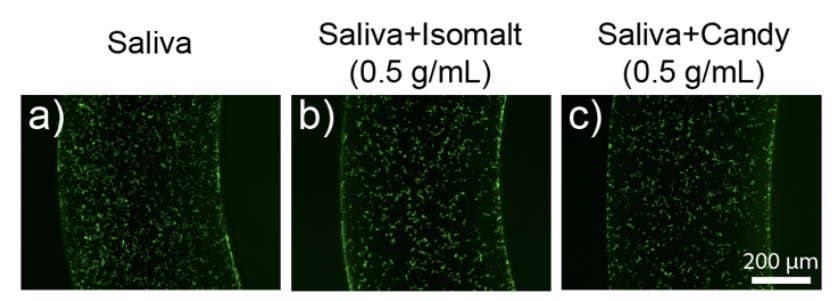

Figure 5. Saliva, isomalt, and other candy ingredients do not interfere with $S$. pyogenes capture on the CandyCollect device. Fluorescence microscopy images of captured S. pyogenes in the device when incubated in solutions containing a) pooled saliva, b) pooled saliva and isomalt, and c) pooled saliva and device candy (composition: isomalt, water, candy oil flavoring, and gel food coloring). Solutions were used to resuspend $S$. pyogenes at a concentration of $1.7 \times 10^{9} \mathrm{CFU} / \mathrm{mL}$, incubated on the CandyCollect device for 2 minutes, and imaged. Images are representative of one independent experiment, with 2 replicates and 3-4 images taken per replicate.

To investigate the variability in dissolving time across different people, we enrolled participants in a pilot feasibility and usability study. The participants were instructed to consume three lollipops that were $0.90-1.10 \mathrm{~g}$, with a diameter of $16 \mathrm{~mm}$, and a thickness of $4 \mathrm{~mm}$ and record the dissolving time (Figure $4 \mathrm{~b}$ ). The average dissolving time across participants is 3.51 mins, with a minimum of 1.25 mins, and the majority of participants completely consumed the candy within $2-4$ mins. The full set of data is available in Table S2. In practice, the required sampling time, which depends on the abundance of a pathogen of interest and the threshold of detection by qPCR, will be evaluated for each pathogen of interest, and the mass of the candy will be adjusted accordingly. We understand that there is personal variability in the amount of time required for the candy to dissolve, and we will adjust the mass and dimensions of the candy so that the minimum dissolving time matches the required collection time for a given pathogen.

During the collection period, the device channel will encounter saliva, bacteria (the analyte of interest) and candy. Isomalt, the primary component of the candy fixed to the device, has been demonstrated to have little impact on bacterial proliferation, ${ }^{20}$ however the candy, in addition to saliva, both have the potential to interfere with the adhesion of $S$. pyogenes to the channels of the device, either through competitive binding or chemical action. To replicate the environment of the device during sample capture in a participant, S. pyogenes was incubated in vitro on the device in mixtures containing pooled saliva, isomalt and/or candy (Fig 5). Stock solutions of isomalt and the device candy were made in phosphate buffered saline (PBS). These solutions were mixed with pooled saliva in a one-to-one ratio for a final concentration of isomalt and candy of $0.5 \mathrm{~g} / \mathrm{mL}$ in their respective solutions in order to maximize the concentration of the isomalt and candy in the saliva. Solutions were used to resuspend $S$. pyogenes and subsequently applied to the devices and incubated. Fluorescence images demonstrate that saliva, isomalt, and the device candy do not impact the adhesion of $S$. pyogenes to the channel of the device (Fig. 5).

\section{Conclusion}

In this work, we developed a saliva sampling device that aims to enable high-quality, comfortable collection of pathogens from saliva in a home or clinical setting. The proposed sampling device shows great potential to (1) effectively capture and concentrate bacteria for further analysis, (2) be effective after storage times of two months, and (3) use candy flavoring as an indicator to optimize the time for bacterial capture. In the future, manufacturing of the proposed device can be scaled 
up using rapid injection molding and testing of the CandyCollect in a clinical setting will provide useful information as to the widespread utility of this device. This technology has the potential to bring sampling technologies into decentralized telemedicine and minimize the need to access a clinic. Furthermore, this CandyCollect platform and advanced sample analysis has great potential for sample collection and diagnosis in other respiratory diseases. Testing of the CandyCollect in a clinical setting will provide useful information as to the widespread utility of this device.

\section{Methods}

\section{Lollipop fabrication}

Lollipop sticks were fabricated out of $2 \mathrm{~mm}$ thick polystyrene sheets (Goodfellow), and an acrylic mold for making the silicone mold was fabricated from a $25.4 \mathrm{~mm}$ thick acrylic (Source One) by CNC milling (Datron Neo). The silicone mold used to cast the lollipops was made by pouring food safe silicone (smooth-sil 940, Smooth-On) into the acrylic mold, and cured for $24 \mathrm{hrs}$ at room temperature. The candy was prepared following hygiene guidance from the Washington State Cottage Food Operations Law (RCW 69.22.040(2b-f(ii-iv)). The study team member who prepared the lollipops trained in food safety, has a Food Worker Card, and wore a mask during food preparation. In brief, the candy was prepared by gradually adding $1000 \mathrm{~g}$ of isomalt to $80 \mathrm{~g}$ of boiling water until the solution reached $\sim 170^{\circ} \mathrm{C}$. Then $3.5 \mathrm{~mL}$ of Chefmaster Super red gel color was added after the solution reached $165^{\circ} \mathrm{C}$. Then $10 \mathrm{~mL}$ of LorAnn Super Strength Strawberry Candy Oil was added after the solution reached $170^{\circ} \mathrm{C}$, and it was removed from the heat. The candy was cooled to room temperature and remelted in small portions for distribution among molds. The silicone mold was placed onto a marble slab to ensure rapid cooling, then the melted candy was poured into each individual lollipop shape until the lollipop was filled. A lollipop stick was placed into the candy, channels facing up, with the circular portion of the polystyrene centered in the candy. The stick was held in place for $15 \mathrm{~s}$, then the next stick was placed. The sticks were pushed back into the candy as needed (using sterilized metal tools) as they set for approximately 5-10 min. Once set, they were carefully removed from the silicone mold and placed on a silicone mat on the marble to finish cooling. After cooling, the lollipops were placed into oriented polypropylene bags (3x4 inch BakeBaking) and heat sealed using an impulse sealer (MetronicModel: FS-200) on setting 3.

\section{Lollipop dissolving time in human subjects}

\section{Participant characteristics}

This study was approved by the University of Washington Institutional Review Board (IRB) under IRB-exempt protocol STUDY00012318. All study procedures were performed after informed consent was obtained. A total of 17 healthy volunteers over the age of 18 years old were recruited via the UW Institute of Translational Health Sciences (ITHS) "participate in research" website and the study team website.

\section{Inclusion and exclusion criteria for participant enrollment}

Inclusion criterion: over the age of 18 years. Exclusion criteria: individuals who are allergic to sugar alcohols or are in vulnerable groups that are pregnant women or individuals residing in a correctional facility. 


\section{Enrollment of participants}

Once potential participants sent us an email sharing their interest in participating, a study team member sent them a link to a pre-screening survey which asked questions about the inclusion and exclusion criteria. Eligible participants were then asked to enter their demographic information, such as their first and last name, shipping address, and contact information. After signing an informed consent form on REDCap (Research Electronic Data Capture, hosted at the ITHS), ${ }^{23}$ participants were enrolled into the study. All identifiable data were securely stored in REDCap and only de-identifiable data were obtained for further analysis.

\section{Human subjects study procedures}

Study participants were enrolled in order to test the general feasibility of the CandyCollects. Each participant was shipped a kit that contained 6 CandyCollects and an instruction card. As directed on the instruction card, each participant consumed one CandyCollect at a time and recorded the total time it took to dissolve the candy on each device. Study data were collected and managed using REDCap.

\section{S. pyogenes culture and imaging}

Todd-Hewitt broth (THY) liquid media preparation

For the THY liquid media, $30 \mathrm{~g}$ of Todd-Hewitt Broth (BD Bacto ${ }^{\text {TM }}$ THY, Fisher Scientific, Cat\# DF0492-17-6) and $2 \mathrm{~g}$ of Yeast Extract (Fisher Scientific, Cat\# NC9796728) were added to 0.8 L distilled water, and dissolved to completion. Additional distilled water was added for a total volume of $1 \mathrm{~L}$. THY liquid media was autoclaved for $30 \mathrm{~min}$, cooled to room temperature and stored at $4^{\circ} \mathrm{C}$.

\section{S. pyogenes maintenance in agar plate}

The $S$. pyogenes was prepared from Streptococcus pyogenes Rosenbach (American Type Culture Collection, ATCC ${ }^{\circledR}, 700294^{\mathrm{TM}}$ ). Freeze-dried S. pyogenes was wetted with $1 \mathrm{~mL}$ liquid media, and transferred the content to another conical tube containing $4.4 \mathrm{~mL}$ of THY liquid media. The concentration of the $S$. pyogenes was $6 \times 10^{8}$ cells $/ \mathrm{mL}$. Then we stored the media with $S$. pyogenes in cryogenic vials (Fisher Scientific, Cat\# 03-374-059) at $-80{ }^{\circ} \mathrm{C}$. To maintain and ensure isolated colonies, S. pyogenes was cultured on Trypticase ${ }^{\mathrm{TM}}$ Soy Agar (TSA II ${ }^{\mathrm{TM}}$ ) with Sheep Blood (BD BBL $^{\text {TM}}$, Fisher Scientific, Cat\# B21239X). A sterile disposable inoculating loop (Fisher Scientific, Cat\# 22-170-201) was dipped in liquid media containing $S$. pyogenes, and streaked along the edge of the agar plate. The plate was then rotated $90^{\circ}$ and the streaking was continued. This was repeated two times more using the same loop. The agar plate was maintained in an incubator at $37^{\circ} \mathrm{C}$ with $5 \%$ carbon dioxide overnight, then stored at room temperature for seven days.

\section{Incubation of S. pyogenes in liquid media}

To ensure a pure culture, fresh $S$. pyogenes in liquid media from agar plates were inoculated one day before the experiment. A pipette tip was used to pick one colony from the agar plate containing viable $S$. pyogenes colonies, and then the pipette tip was stirred in $7 \mathrm{~mL}$ THY liquid media in a sterile $14 \mathrm{~mL}$ snap-cap round bottom polystyrene tube (Falcon®, Corning, Product\# 352001). $S$. pyogenes was incubated at $37^{\circ} \mathrm{C}$ with $5 \%$ carbon dioxide in the incubator overnight. 
Fixing and staining of S. pyogenes

The device was rinsed with PBS and fixed with 4\% Paraformaldehyde (PFA) for 15 minutes. To remove excess PFA, the device was rinsed with PBS. $50 \mu \mathrm{L}$ of Alexa FluorTM 488 Wheat Germ Agglutinin (WGA, Invitrogen TM, W1126, Fisher Scientific, Pittsburgh, PA) at 1: 500 dilution (v/v) was added to the channel and incubated for 30 minutes at room temperature in the absence of light. The device was rinsed with PBS to remove excess WGA. Then the device was stored in centrifuge tubes containing PBS. The centrifuge tubes were covered with aluminum foil and stored at $4{ }^{\circ} \mathrm{C}$ before imaging.

Imaging

Fluorescent images of S. pyogenes were obtained on a Zeiss Axiovert 200 with a $10 \times(0.30$ NA) magnification coupled with Axiocam 503 mono camera (Carl Zeiss AG, Oberkochen, Germany). Multiple channel regions (four to five) were chosen randomly from each device to take images for confirming no bias on a specific region. For all images, the contrast was adjusted uniformly using Fiji (ImageJ) software.

\section{Cotton swab scratching assay}

$50 \mu \mathrm{l}$ of S. pyogenes suspension at the concentration of $1 \times 10^{\wedge} 9 \mathrm{CFU} / \mathrm{mL}$ were applied on each CandyCollect device and the device without channels (flat surface) of identical design and incubated for $10 \mathrm{~min}$. The devices were rinsed once with PBS and excess liquid was drained with KimWipes. Then strokes were made on the surface of each device using a cotton swab, to mimic scraping by the tongue. Control devices were not touched by cotton swabs. To evaluate the effect of cotton swab scratching on bacteria binding, the bacteria on the device were stained and imaged following protocol stated above.

\section{Plasma treatment shelf life experiments}

\section{Oxygen plasma treatment}

The devices were plasma treated by Zepto LC PC Plasma Treater (Diener Electronic GmbH, Ebhausen, Germany). The chamber was pumped down to a pressure of $0.20 \mathrm{mbar}$, gas was supplied ( 0.25 mbar for 2 minutes), power enabled ( $70 \mathrm{~W}$ for 5 minutes). After plasma treatment, the devices were stored in OmniTrays (Thermo Scientific ${ }^{\mathrm{TM}}$ Nunc $^{\mathrm{TM}}$ OmniTray ${ }^{\mathrm{TM}}$, Fisher Scientific, Cat\# 12565-296) under room temperature for zero day (control group), three days, one week, two weeks, two months with $n=3$ replicated devices before the experiment. For time points less than one month, devices were treated in descending order (i.e., two-week devices treated first, followed by one-week devices 7 days later) allowing for the two-week, one-week, and three-day devices to be tested on the same day. The two-months devices were tested on a different day. The zero day (control group) was done for every experiment set.

\section{S. pyogenes experiments to determine shelf life}

The concentration of $S$. pyogenes was confirmed through Visible 721-Vis Spectrophotometer (vinmax) at a wavelength of $600 \mathrm{~nm}$. S. pyogenes in liquid media was centrifuged in $1 \mathrm{~mL}$ at $10,000 \mathrm{rpm}$ for 10 minutes. $S$. pyogenes was re-suspended in the THY liquid media at a density of $10^{9} \mathrm{CFU} / \mathrm{mL}$. $50 \mu \mathrm{L}$ of the suspended $S$. pyogenes was pipetted into the device channel and incubated for 10 minutes (unless otherwise specified). The bacteria on the device were stained and imaged following protocol stated above. 


\section{Saliva and candy interference experiment}

Candy-bacteria stock solutions

A stock solution of isomalt was made at a $1.0 \mathrm{~g} / \mathrm{mL}$ concentration in phosphate buffered saline (PBS). This solution was mixed with pooled saliva in a one-to-one ratio (final isomalt concentration $0.5 \mathrm{~g} / \mathrm{mL}$ ). This process was repeated with the device candy (composition: isomalt, water, candy oil flavoring, and gel food coloring). These solutions were used to resuspend $\mathrm{S}$. pyogenes at a concentration of $1.7 \times 10^{9} \mathrm{CFU} / \mathrm{mL}$.

\section{Elution of $S$. pyogenes from dried samples on devices}

$S$. pyogenes was resuspended in the mixture of pooled saliva and PBS (1:1) at a final concentration of $1 \times 10^{\wedge} 9 \mathrm{CFU} / \mathrm{mL}$. Each device was incubated with $50 \mu \mathrm{L}$ of the bacterial suspension for $10 \mathrm{~min}$, followed by drying in a biosafety cabinet for $10 \mathrm{~min}$. Then the devices were transferred to $15 \mathrm{ml}$ round bottom tubes containing $300 \mu \mathrm{L}$ of DNAzol ${ }^{\mathrm{TM}}$ Reagent (Invitrogen, Cat\#10503-027). The tubes were vortexed to dislodge bacteria from the devices. To check the effect of elution, the bacteria on the device were stained and imaged following protocol stated above.

\section{Acknowledgements}

This work was supported by a Washington Research Foundation (WRF) Phase 1 Grant, the NIH grant (R21AI147502-02), the National Institute of General Medical Sciences award number R35GM128648 (for some of the in-lab developments and in vitro experiments), the Society for Laboratory Automation and Screening (SLASFG2020, UNL), the David and Lucile Packard Foundation, and the University of Washington. REDCap at UW ITHS is supported by the National Center For Advancing Translational Sciences of the National Institutes of Health under Award Number UL1 TR002319. The content is solely the responsibility of the authors and does not necessarily represent the official views of the Society for Laboratory Automation and Screening or the National Institutes of Health.

\section{Conflicts of interest}

A.B.T. has ownership in Stacks to the Future, LLC and E.B. has ownership in Stacks to the Future, LLC, Tasso, Inc., and Salus Discovery, LLC. However, this research is not related to these companies.

\section{References}

1. H. J. Zar and T. W. Ferkol, Journal, 2014, DOI: 10.1002/ppul.23030.

2. E. A. Simoes, T. Cherian, J. Chow, S. A. Shahid-Salles, R. Laxminarayan and T. J. John, Disease Control Priorities in Developing Countries. 2nd edition, 2006.

3. S. T. Shulman, A. L. Bisno, H. W. Clegg, M. A. Gerber, E. L. Kaplan, G. Lee, J. M. Martin and C. Van Beneden, Clinical Infectious Diseases, 2012, 55, e86-e102.

4. R. N. Piasio, N. Turner, A. Wheeler, Methods and devices for testing saliva. 2007 (WO2008073895A3).

5. L. E. Norton, B. R. Lee, L. Harte, K. Mann, J. G. Newland, R. A. Grimes and A. L. Myers, Pediatrics, 2018, 142.

6. $\quad$ R. Uhl James, R. Patel and E. Munson, Journal of Clinical Microbiology, 2016, 54, 815815.

7. P. Weisenseel and J. C. Prinz, Archives of Dermatological Research, 2005, 296, 573-576. 
8. Y. H. Tsai, P. H. Chen, P. A. Yu, C. L. Chen, L. T. Kuo and K. C. Huang, Int J Infect Dis, 2019, 81, 73-80.

9. A. Ahmed, J. V. Rushworth, J. D. Wright and P. A. Millner, Analytical Chemistry, 2013, 85, 12118-12125.

10. N. Malinowska, W. Białobrzeska, T. Łęga, K. Pałka, K. Dziąbowska, S. Żołędowska, E. Czaczyk, K. Pala and D. Nidzworski, Sensors, 2020, 20.

11. A. K. Jarmusch, V. Pirro, K. S. Kerian and R. G. Cooks, Analyst, 2014, 139, 4785-4789.

12. M. A. Fragoso, L. Manning and L. D. Frenkel, Pediatr Infect Dis J, 1989, 8, 845-847.

13. M. Kaitz, R. Sabato, I. Shalev, R. Ebstein and D. Mankuta, Developmental Psychobiology, 2012, DOI: 10.1002/dev.20580.

14. A. Shelburne Samuel, C. Granville, M. Tokuyama, I. Sitkiewicz, P. Patel and M. Musser James, Infection and Immunity, 2005, 73, 4723-4731.

15. G. DeMuri and E. R. Wald, Clinical Pediatrics, 2020, DOI: 10.1177/0009922820920936.

16. G. Karthikeyan and L. Guilherme, Lancet, 2018, 392, 161-174.

17. S. L. Barker and P. J. LaRocca, Journal of tissue culture methods, 1994, 16, 151-153.

18. A. Vesel, M. Mozetic, M. Jaganjac, L. Milkovic, A. Cipak and N. Zarkovic, The European Physical Journal - Applied Physics, 2011, 56, 24024.

19. M. J. Lerman, J. Lembong, S. Muramoto, G. Gillen and J. P. Fisher, Tissue Engineering Part B: Reviews, 2018, 24, 359-372.

20. A. Zumbé, A. Lee and D. Storey, British Journal of Nutrition, 2001, 85, S31-S45.

21. K. Tiefenbacher and K. Tiefenbacher, Wafer and Waffle, 2017, 123-225.

22. O. M. Ba, P. Marmey, K. Anselme, A. C. Duncan and A. Ponche, Colloids and Surfaces B: Biointerfaces, 2016, 145, 1-7.

23. P. A. Harris, R. Taylor, R. Thielke, J. Payne, N. Gonzalez and J. G. Conde, Journal of Biomedical Informatics, 2009, 42, 377-381. 